\title{
A Rapidly Progressing Pleural Effusion within 24 Hours: A Case Report
}

\author{
Bassem Alhariri ${ }^{1}$, Ayisha Ameen ${ }^{1}$, and Abdulqadir Nashwan ${ }^{1}$ \\ ${ }^{1}$ Hamad Medical Corporation
}

October 22, 2021

\begin{abstract}
Patients with pleural effusion are mostly presenting with shortness of breath and pleuritic chest pain. This report describes a case of PE who presented with left shoulder pain and was found to have rapidly accumulating massive effusion within 24 hours of presentation. Thoracocentesis was performed a showed an exudative picture
\end{abstract}

Short title: A Rapidly Progressing Pleural Effusion

Bassem N. Alhariri ${ }^{1}$, Ayisha Ameen ${ }^{1}$, Abdulqadir J. Nashwan ${ }^{2}$

1. Department of Medicine, Hazm Mebaireek General Hospital (HMGH), Hamad Medical Corporation (HMC), Doha, Qatar

2. Department of Nursing, Hazm Mebaireek General Hospital (HMGH), Hamad Medical Corporation (HMC), Doha, Qatar

Corresponding author:

Mr. Abdulqadir J. Nashwan

anashwan@hamad.qa

Tel: $(+974) 40240487$

Mob: (+974) 66473549

P.O.Box 3050 Doha, Qatar

\section{Abstract}

Patients with pleural effusion are mostly presenting with shortness of breath and pleuritic chest pain. This report describes a case of $\mathrm{PE}$ who presented with left shoulder pain and was found to have rapidly accumulating massive effusion within 24 hours of presentation. Thoracocentesis was performed a showed an exudative picture.

Keywords: massive pleural effusion; thoracocentesis; exudative effusion; complicated parapneumonic effusion

\section{Key Clinical Message}

Patients can present with atypical symptoms on admission but may develop massive parapneumonic pleural effusion within a short time that would need definite management via urgent chest tube placement.

\section{BACKGROUND}


A pleural effusion (PE) occurs due to a disturbance in the balance between pleural fluid (PF) formation and removal. ${ }^{1}$ It does not arise as a disease by itself; instead is an outcome of underlying pathology. Therefore, diagnosing PE requires a physician to determine its cause to treat PE appropriately. Still, in nearly $20 \%$ of cases, the etiology of PE remains unclear. Therefore, a careful history with good clinical examination should aid in diagnosing, especially in patients with atypical presentations. Thoracocentesis is the crucial step to determine the various etiologies. Considering the PF biochemistry, cytology, and signs and symptoms; PE diagnosis can be established in the vast majority of the cases. ${ }^{2} \mathrm{PF}$ analysis can be classified as exudative or transudative. Treating the underlying cause is the typical management strategy for transudative effusions.

Massive PE refers to an effusion that fills almost all the hemithorax. A prospective study was conducted to establish the most common causes of pleural effusions. Malignant effusion is the leading cause of massive effusion, followed by PE secondary to cirrhosis. On the other hand, massive pleural effusions were far less likely to be caused by congestive heart failure or infections. ${ }^{3}$ The study added to the growing body of evidence that, whatever the source, a substantial, refractory PE, whether transudate or exudate, symptomatic relief could be established by drainage.

\section{CASE PRESENTATION}

A 44-year-old ex-smoker with no known co-morbidities arrived at our emergency room with a one-week complaint of left-sided chest discomfort and left shoulder pain. The pain was gradual in onset and continuous in nature, radiating to the shoulder. The pain increased while deep breathing and on movements. The pain was not relieved by any medications. The patient complained of fever at night and dry cough, and some loss of appetite but no history of shortness of breath, productive cough, no night sweats, no history of significant weight loss. He denied known TB contact and any history of TB. No other history of any traumatic event (Table 1). The patient was afebrile on examination, with a heart rate (HR) of 90 beats per minute and blood pressure (BP) of $130 / 73 \mathrm{mmHg}$, respiratory rate (RR) of 19 breaths per minute, and an oxygen saturation $\left(\mathrm{O}_{2}\right.$ sat $)$ of 100 percent on room air. No clubbing or supraclavicular lymphadenopathy On examination of the respiratory system, there was no visible deformity, no tenderness on palpation, the percussion note was dull on the lower left side of the chest, and the percussion note was dull on the on auscultation decreased air entry on the left side with left basal crackles.

Examination of the left shoulder joint showed no tenderness and a normal range of movements. The remaining systemic examination, including cardiovascular, neurological, and gastrointestinal exam, was unremarkable. A chest x-ray (CXR) (Figure 1) was performed, which showed a mild/moderate amount of left-sided PE with underlying atelectasis. The diagnosis of left-sided PE was made, and the patient was started on antibiotics empirically as a case of parapneumonic effusion. Diagnostic PF aspiration was done under septic conditions, and workup was sent for PF analysis to determine the cause of the effusion without typical respiratory symptoms. The patient was kept on isolation is suspicion of TB. Within the next 24 hours; the patient developed worsening chest pain on the left side, shortness of breath, and desaturation. A repeated CXR (Figure 2) was performed and compared with the previous CXR; there is a considerable interval increasing amount of PE with collapse/consolidation left lung. In the account of thoracocentesis done the previous day, traumatic hemothorax was suspected but was ruled out as there was no drop in hemoglobin. The diagnosis of massive PE on the left side that had rapidly progressed over 24 hours, with worsening chest symptoms, was made. An urgent chest drain was inserted under ultrasound guidance, and drainage was done. Following this, a CT chest was done (Figure 3), and it showed improvement in the PE and no evidence of empyema or abscess formation. The patient started feeling better with the chest drain in place, and he drained. He improved clinically, and the inflammatory markers improved from admission. The PF analysis showed an exudative picture with a neutrophilic predominance (Table 2). PF cultures were negative. Work up for TB and malignancy were negative.

On Day 9, after the chest tube was inserted, the drainage of fluid was nil. A repeat CXR was done, and it showed resolution of $\mathrm{PE}$, the chest tube was removed, and the patient was discharged on antibiotics for a total of 21 days, and an outpatient follow-up appointment with the pulmonologist was given. 


\section{DISCUSSION}

The pleural sac is a compartment typically filled with a thin layer of fluid known as pleural fluid, which acts as a lubricant and helps reduce friction between the parietal and visceral pleural lining surfaces during respiration. ${ }^{4}$ An accumulation of fluid occurs because of an imbalance in the rate of production and drainage of fluid due to changes in many local factors. ${ }^{4}$

The typical presentation of a case of PE may include the pleuritic type of chest pain, which indicates inflammation of the pleura, with the pain increasing on movement and respiration with some associated shortness of breath and dry cough. In contrast, the patient presented with only left-sided shoulder pain with no respiratory symptoms in this case. As a clinician, the underlying PE may present with these atypical symptoms and should not be simply mistaken for musculoskeletal pain. PE should be on the top of the list of differentials. An underlying undiagnosed rapidly accumulating PE can, in turn, lead to rapid deterioration of the patient.

The first step in determining the cause of a PE in cases where clinical history does not correlate, but PE is evident on clinical imaging is to perform a diagnostic thoracocentesis; this will help a clinician to differentiate if the $\mathrm{PE}$ is transudative or exudative. However, diagnostic thoracentesis is not required in patients with a small quantity of $\mathrm{PF}(<500 \mathrm{cc})$ and a definite diagnosis (e.g., viral pleurisy) or in patients with heart failure (HF), obvious on clinical examination but no unusual findings. ${ }^{5}$

In patients with an exudative PE, like the case we are discussing here, Further evaluation is needed to determine the underlying pathology affecting the pleura leading to the PE. Lactic Acid Dehydrogenase $(\mathrm{LDH})$ and serum protein levels in the PF and serum are used to confirm an exudative effusion diagnosis. The Light's criteria are the most widely used criterion for distinguishing between exudative and transudative effusions. ${ }^{6,7}$

According to the lights criteria; the following can be calculated:

- Ratio between PF protein and serum protein >0.5;

- Ratio between PF LDH and serum LDH >0.6;

- $\mathrm{LDH}$ in PF is more than two-third of the upper limit of serum LDH.

When one of the three conditions is satisfied, the effusion is exudative, as in this example. A variety of conditions can cause exudative effusions, and their care can be difficult since they frequently necessitate surgical interventions in addition to the medical management. ${ }^{8}$

What strikes the most, in this case, is that how the patient initially on presentation had mild PE and needed only medical treatment for his underlying asymptomatic pneumonia. However, within the next 24 hours, the patient started to reaccumulate fluid in his pleural space, which leads to his sudden clinical deterioration, which required the urgent need for chest tube placement and drainage under interventional radiology guidance.

Massive pleural effusions are a common manifestation of pulmonary tuberculosis, ${ }^{9}$ or an underlying malignancy. It has been reported in malignancies of different origins, the most frequent being ovarian carcinoma, sarcomas, mesotheliomas, and pancreatic adenocarcinomas. Some cases of massive PE, although rare are reported even in patients with pancreatic pleural fistulas, alcohol-induced pancreatitis and sarcoidosis. ${ }^{10}$

In our patient, the rapidly developing and reaccumulating PF raised the suspicion for any underlying malignancy or TB as statistics prove it to be the most common pathological cause, but the diagnostic pleural tapping in this patient ruled out these causes and the workup for any other potential cause was not supported by further investigations that were done.

Therefore, the diagnosis of massive parapneumonic effusion was made do the neutrophilic predominant picture of the thoracocentesis, which was complicated with drainage via a chest tube, although this is rare in literature to accumulate within a short period of time rapidly. 


\section{CONCLUSION}

Clinicians and pulmonologists must be aware of the potential atypical presentations of PE for timely patient management. A high index of suspicion must be maintained for patients with left shoulder pain who can have an underlying collection of fluid in their pleural space, leading to rapid deterioration due to desaturation.

\section{Abbreviations}

PE: Pleural Effusion

PF: Pleural Fluid

LDH: Lactic Acid Dehydrogenase

\section{Declarations}

\section{Ethics approval and consent to participate}

The article describes a case report. Therefore, no additional permission from our Ethics Committee was required.

\section{Consent for publication}

The consent for publication was obtained.

\section{Availability of data and material}

All data generated or analyzed during this study are included in this published article.

\section{Competing interests}

The authors declare that they have no competing interests.

\section{Funding}

This study was not funded.

\section{Authors' contributions}

BNA, AYA, AJN: Data Collection, Literature Search, Manuscript Preparation

All authors read and approved the final manuscript

\section{Acknowledgments}

We thank the patient for allowing us to share his case. Open Access funding provided by the Qatar National Library

\section{References}

1. Karkhanis VS, Joshi JM. Pleural effusion: diagnosis, treatment, and management. Open access emergency medicine: OAEM. 2012;4:31.

2. Collins TR, Sahn SA. Thoracocentesis: clinical value, complications, technical problems, and patient experience. Chest.1987;91(6):817-822.

3. Jiménez D, Díaz G, Gil D, et al. Etiology and prognostic significance of massive pleural effusions. Respiratory medicine.2005;99(9):1183-1187.

4. Koppurapu V, Meena N. A review of the management of complex para-pneumonic effusion in adults. Journal of thoracic disease.2017;9(7):2135.

5. Kasper D, Fauci A, Hauser S, Longo D, Jameson J, Loscalzo J.Harrison's principles of internal medicine, 19e. Vol 1: Mcgraw-hill New York, NY, USA:; 2015. 
6. Light RW, Macgregor MI, Luchsinger PC, BALL JR WC. Pleural effusions: the diagnostic separation of transudates and exudates. Annals of internal medicine. 1972;77(4):507-513.

7. Light RW. The Light criteria: the beginning and why they are useful 40 years later. Clinics in chest medicine. 2013;34(1):21-26.

8. Jany B, Welte T. Pleural effusion in adults - etiology, diagnosis, and treatment. Deutsches Ärzteblatt International.2019;116(21):377.

9. Soe Z, Shwe WH, Moe S. A study on tuberculous pleural effusion.International Journal of Collaborative Research on Internal Medicine 85 Public Health. 2010;2(3):0-0.

10. Sherani K, Alnuaimi A, Vakil A, Cervellione K, Kulsum U, Shalonov A. Massive Pleural Effusion as an Initial Manifestation of Sarcoidosis. Chest. 2016;149(4):A225.

\section{Tables and Figures}

Table 1. Laboratory investigations upon admission

Table 2. Pleural fluid analysis

Figure 1. left-sided pleural effusion with underlying atelectasis.

Figure 2. a large amount of pleural effusion on the left side with the collapse of lung parenchyma

Figure 3. mild pleural effusion on the left side no evidence of collection

\section{Hosted file}

Fig1.docx available at https://authorea.com/users/442438/articles/542713-a-rapidlyprogressing-pleural-effusion-within-24-hours-a-case-report

\section{Hosted file}

Fig2.docx available at https://authorea.com/users/442438/articles/542713-a-rapidlyprogressing-pleural-effusion-within-24-hours-a-case-report

\section{Hosted file}

Fig3.docx available at https://authorea.com/users/442438/articles/542713-a-rapidlyprogressing-pleural-effusion-within-24-hours-a-case-report 